\title{
Low serum estradiol levels are related to Mycobacterium avium complex lung disease: a cross-sectional study
}

Yoshifumi Uwamino ${ }^{1,2}$, Tomoyasu Nishimura ${ }^{3 *}$, Yasunori Sato ${ }^{4}$, Eiko Tamizu ${ }^{2}$, Takanori Asakura ${ }^{5}$, Shunsuke Uno ${ }^{2}$, Masaaki Mori ${ }^{3}$, Hiroshi Fujiwara ${ }^{2}$, Makoto Ishii ${ }^{5}$, Hiroshi Kawabe ${ }^{6}$, Mitsuru Murata ${ }^{1}$ and Naoki Hasegawa ${ }^{2}$

\begin{abstract}
Background: The risk factors for Mycobacterium avium complex lung disease (MAC-LD) are not well known. We hypothesized that low serum estradiol (E2) levels are related to MAC-LD as most patients with MAC-LD are postmenopausal women.

Methods: This cross-sectional study compared patients with MAC-LD and healthy controls. Study subjects were postmenopausal women aged 65 years or younger. Serum testosterone, dehydroepiandrosterone sulfate (DHEA-S), and E2 levels were measured and categorized as high or low based on median levels. We performed multivariate analysis, receiver operating characteristic (ROC) curve analysis, and age- and body mass index (BMI)-matched subgroup analysis to evaluate the association between low serum E2 levels and MAC-LD.

Additionally, using blood samples obtained for other clinical studies, the levels of sex steroid hormones were compared between age- and BMI-matched MAC-LD and bronchiectasis female patients without non-tuberculosis mycobacterial infections (non-NTM BE).

Results: Forty-two patients with MAC-LD and 91 healthy controls were included. The median E2 $(2.20 \mathrm{pg} / \mathrm{mL} \mathrm{vs}$. $15.0 \mathrm{pg} / \mathrm{mL}, p<0.001)$, testosterone $(0.230 \mathrm{ng} / \mathrm{L}$ vs. $0.250 \mathrm{ng} / \mathrm{L}, p=0.005)$, and DHEA-S $(82.5 \mu \mathrm{g} / \mathrm{dL}$ vs. $114.0 \mu \mathrm{g} / \mathrm{dL}$, $p<0.001)$ levels were lower in the MAC-LD group than in the control group. Multivariate analysis revealed that low serum E2 (adjusted odds ratio $=34.62,95 \%$ confidence interval $=6.02-199.14$ ) was independently related to MACLD, whereas low DHEA-S and testosterone were not. ROC analysis illustrated a strong relationship between low serum E2 levels and MAC-LD (area under the curve $=0.947$, 95\% confidence interval $=0.899-0.995$ ). Even the ageand BMI-matched subgroup analysis of 17 MAC-LD patients and 17 healthy controls showed lower serum E2 in MAC-LD patients than in healthy controls. Additionally, serum E2 levels of 20 MAC-LD patients were lower than plasma E2 levels of 11 matched non-NTM BE patients (1.79 pg/mL vs. $11.0 \mathrm{pg} / \mathrm{mL}, p<0.001)$.
\end{abstract}

Conclusions: Low serum E2 levels were strongly related to MAC-LD in postmenopausal women.

Keywords: Estradiol, Mycobacterium avium complex, Postmenopausal women

\footnotetext{
* Correspondence: tnishimura@keio.jp

${ }^{3}$ Keio University Health Center, 35 Shinanomachi, Shinjuku-ku, Tokyo

160-8582, Japan

Full list of author information is available at the end of the article
}

(c) The Author(s). 2019 Open Access This article is distributed under the terms of the Creative Commons Attribution 4.0 International License (http://creativecommons.org/licenses/by/4.0/), which permits unrestricted use, distribution, and reproduction in any medium, provided you give appropriate credit to the original author(s) and the source, provide a link to the Creative Commons license, and indicate if changes were made. The Creative Commons Public Domain Dedication waiver (http://creativecommons.org/publicdomain/zero/1.0/) applies to the data made available in this article, unless otherwise stated. 


\section{Background}

The incidence of pulmonary nontuberculous mycobacterial (NTM) infection continues to increase globally [13]. Although Mycobacterium avium complex (MAC) lung disease (MAC-LD) accounts for approximately 80 and $90 \%$ of all cases of pulmonary NTM infection in the United States and Japan, respectively, MAC-LD is a chronic and refractory respiratory infection with unclear pathogenesis and unknown risk factors.

A number of epidemiological studies have revealed that patients with MAC-LD are predominantly elderly and postmenopausal women $[4,5]$. We previously estimated the MAC infection rate in a healthy population by measuring immunoglobulin (Ig) G antibody titers against the MAC-specific lipid antigen glycopeptidolipid (GPL) [6]. Interestingly, the antibody prevalence in middle-aged to elderly women was significantly higher than that in men or younger women, indicating that middle-aged and elderly women were more likely to be infected with MAC. On the basis of these findings, decreases in sex hormone levels, especially estradiol (E2), after menopause might represent a risk factor for MACLD. However, no previous study has verified this hypothesis.

Therefore, we conducted a cross-sectional study to determine whether low serum E2 levels are related to MAC-LD.

\section{Methods}

\section{Study population}

We conducted a cross-sectional study comparing postmenopausal women with MAC-LD with healthy postmenopausal women at a tertiary care center in Japan. Data and samples from outpatients with MAC-LD were obtained between May 2016 and April 2017 from Keio University Hospital, one of the largest referral centers in Tokyo. The hospital treats approximately 400 outpatients annually with MAC-LD in its infectious disease and pulmonary disease clinics. In total, 193 women with MAC-LD provided written informed consent for study participation. The diagnosis of MAC-LD was based on 2007 American Thoracic Society/Infectious Diseases Society of America criteria [7]. Meanwhile, data and samples from healthy controls were obtained through annual health examinations of the faculty and staff of Keio University held at the Mita (Tokyo), Hiyoshi (Kanagawa), and Shonan Fujisawa (Kanagawa) campuses between September 2015 and November 2015. In total, 383 healthy female faculty and staff, who were not healthcare workers, provided written informed consent for study participation.

We obtained a 9-mL blood sample and clinical information through questionnaires and medical records from each study participant. To analyze sex steroid hormones, we included postmenopausal participants aged 65 years or younger because the levels of sex steroid hormones, especially E2, fluctuate considerably with the menstrual cycle and decline with aging. Because 65 years of age is the retirement age for faculty and staff at Keio University, all healthy controls were aged 65 years or younger. Consequently, we excluded patients with MAC-LD who were older than 65 years.

Participants were also excluded if their information was incomplete regarding menopause and the use of sex hormone-based medications such as oral contraceptives and if they used sex-hormone based medications. Additionally, healthy controls with histories of MAC-LD or chest X-ray findings compatible with MAC-LD were excluded. To exclude healthy controls with latent MAC infection without clinical symptoms or chest X-ray abnormality, we measured anti-GPL core IgA antibody titers using a Capilia MAC Ab ELISA kit (TAUNS Laboratories, Inc. Izunokuni, Shizuoka, Japan). GPL is a specific antigen that constitutes the cell wall of MAC; therefore, anti-GPL antibodies are specific for MAC infection [8]. A cutoff value of $0.3 \mathrm{U} / \mathrm{mL}$ denoted positivity for anti-GPL core IgA antibodies, [9] and healthy controls with titers exceeding this cutoff were excluded.

To compare the demographic characteristics between the MAC-LD and control groups, we obtained information on age, body mass index (BMI), age at first menstruation, age at menopause, years after menopause, smoking history, and history of tuberculosis, human immunodeficiency virus (HIV) infection, immunosuppression (immunodeficiency or use of immunosuppressive medication), and chronic diseases such as hypertension, diabetes, dyslipidemia, and osteoporosis. Information on age, BMI, history of tuberculosis, HIV, immunosuppression, and history of chronic diseases was obtained from medical and health examination records. Histories of chronic diseases were compiled using medication histories. The ages at first menstruation and menopause, and smoking histories were obtained using a questionnaire. The number of years after menopause was calculated from current age and age at menopause.

To determine if the sex steroid hormone levels were associated specifically with MAC-LD, but not other chronic lung diseases, we conducted an additional study to compare serum sex steroid hormone levels of MACLD patients with plasma sex steroid hormone levels of bronchiectasis (BE) patients without NTM infections (non-NTM BE). Pooled blood samples previously obtained for two clinical studies were used. Samples of female MAC-LD patients above 55 years of age, who were diagnosed with MAC-LD based on the 2007 American Thoracic Society/Infectious Diseases Society of America criteria, [7] were selected from pooled serum samples obtained for the "NTM Biomarker Study," which 
included serum samples of NTM patients of Keio University Hospital. Meanwhile, samples of female nonNTM BE patients were selected from pooled plasma samples obtained for the "NTM Genome-Wide Analysis Study," which included plasma samples of NTM patients and BE patients of Keio University Hospital. The following characteristics defined non-NTM BE: 1) clinically diagnosed as BE by respiratory physicians based on CT examination, 2) all previous sputum Mycobacteria culture and smear were negative (at least 2 negative culture results were required), 3) anti-GPL core antibody was not detected.

Because of the small number of non-NTM BE patients, we selected age- (difference within a range of 3 years) and BMI- (difference within a range of $3 \mathrm{~kg} / \mathrm{m}^{2}$ ) matched MAC-LD patients and non-NTM BE patients at a ratio of 2:1. Among the matched pairs, a pair of MAC-LD and non-NTM BE patients was excluded if one of the patients was taking oral steroids or sex hormone medication or had a history of ovariectomy or hysterectomy, which might affect sex hormone production.

\section{Measurement of serum or plasma levels of sex hormones}

We measured serum E2 and testosterone levels using a chemiluminescent immunoassay (CLIA) and measured dehydroepiandrosterone sulfate (DHEA-S) levels using a chemiluminescent enzyme immunoassay. If serum E2 levels were under the limit of detection for CLIA (10 pg/ $\mathrm{mL})$, a further measurement was performed via liquid chromatography-tandem mass spectrometry.

\section{Statistical methods}

For baseline variables, summary statistics were constructed employing frequencies and proportions for categorical data, and medians and interquartile ranges for continuous variables. We compared patient characteristics using Fisher's exact test for categorical outcomes and the Mann-Whitney $U$ test for continuous variables.

Specifically in the analyses comparing MAC-LD patients with healthy controls, to assess factors independently associated with MAC-LD (i.e., age, BMI, years after menopause, E2, testosterone, and DHEA-S), we additionally performed logistic regression analyses via the simultaneous method. For the analyses, we categorized the serum hormone level of each participant as high or low based on the median hormone level of the study population. Thus, the logistic regression model consisted of three continuous (age, BMI, and years after menopause) and three categorical (low or high E2, testosterone, and DHEA-S) values.

Additionally, to assess the strength of the relationship between MAC-LD and low serum E2 levels, we performed receiver operating characteristic (ROC) curve analysis and calculated the area under the curve (AUC). A $p$-value $<0.05$ was considered to be statistically significant. All statistical analyses were performed using SPSS version 25 (IBM, Chicago, IL, USA).

\section{Age- and BMI-matched subgroup analysis}

Additionally, we performed an age- and BMI-matched subgroup analysis of MAC-LD patients and healthy controls, because age and BMI could be strong confounding factors of sex steroid hormones. We selected age- (exact match) and BMI- (difference within a maximum range of $2 \mathrm{~kg} / \mathrm{m}^{2}$ ) matched MAC-LD patients and healthy controls at a ratio of 1:1. Using this matched subgroup, we performed ROC curve analysis.

\section{Results}

\section{Study population}

From the original dataset, 42 patients with MAC-LD and 101 healthy controls fulfilled the inclusion criteria. Three healthy controls using hormone medications were excluded. In addition, seven healthy controls with histories of MAC-LD or anti-GPL core IgA antibody positivity were excluded. Thus, the analysis ultimately included 42 patients with MAC-LD and 91 healthy controls.

The patients with MAC-LD were significantly older than the healthy controls (61 years vs. 56 years, $p<$ 0.001), whereas BMI was significantly lower in the MAC-LD group $\left(19.14 \mathrm{~kg} / \mathrm{m}^{2}\right.$ vs. $\left.22.18 \mathrm{~kg} / \mathrm{m}^{2}, p<0.001\right)$. There were no significant differences regarding the age at first menstruation, menopausal age, smoking history, or history of tuberculosis and chronic diseases between the two groups (Table 1). There were no patients with HIV or immunosuppression except for one patient in the MAC-LD group taking methotrexate for rheumatoid arthritis.

\section{Serum levels of sex hormones}

Serum E2 $(2.20 \mathrm{pg} / \mathrm{mL}$ vs. $15.0 \mathrm{pg} / \mathrm{mL}, p<0.001)$, testosterone $(0.23 \mathrm{ng} / \mathrm{L}$ vs. $0.25 \mathrm{ng} / \mathrm{L}, p=0.005)$, and DHEA-S $(82.5 \mu \mathrm{g} / \mathrm{dL}$ vs. $114.0 \mu \mathrm{g} / \mathrm{dL}, p<0.001)$ levels were significantly lower in the MAC-LD group than in the control group (Fig. 1).

\section{Low serum E2 levels were independently related to MAC- LD}

According to the logistic regression analysis, age and low serum E2 levels were independently related to MAC-LD (Table 2). As expected, the number of years after menopause was strongly correlated with age (Spearman's rank correlation coefficient; $\mathrm{r}=0.727, p<$ $0.001)$; therefore, the number of years after menopause was not included in the logistic regression analysis. To determine the strength of the correlation between serum E2 levels and MAC-LD, ROC curve analysis was 
Table 1 Demographic characteristics of the study subjects

\begin{tabular}{|c|c|c|c|}
\hline & MAC-LD $(n=42)$ & Control $(n=91)$ & $p$-value ${ }^{\dagger}$ \\
\hline Age, years, median (IQR) & $61(59-64)$ & $56(54-59)$ & $<0.001$ \\
\hline BMI, median (IQR) & $19.14(17.85-20.58)$ & $22.18(19.68-24.53)$ & $<0.001$ \\
\hline Age at menopause, years, median (IQR) & $50.5(50-53)$ & $51(49-53)$ & 0.798 \\
\hline Age at first menstruation, years, median (IQR) & $12.5(12-13.75)$ & $12(12-13)$ & 0.383 \\
\hline Years after menopause, years, median (IQR) & $11.5(8-12.75)$ & $5(2-8.25)$ & $<0.001$ \\
\hline History of tuberculosis, n (\%) & $1(2.4)$ & $4(4.4)$ & 1.000 \\
\hline Chronic diseases, n (\%) & $10(23.8)$ & $32(35.2)$ & 0.231 \\
\hline Hypertension, $\mathrm{n}(\%)^{*}$ & $3(7.1)$ & $16(17.5)$ & 0.181 \\
\hline Diabetes mellitus, $\mathrm{n}(\%)^{*}$ & $1(2.4)$ & $3(3.3)$ & 1.000 \\
\hline Dyslipidemia, n (\%)* & $2(4.8)$ & $17(18.7)$ & 0.035 \\
\hline Osteoporosis, n (\%)* & $5(11.9)$ & $3(3.3)$ & 0.108 \\
\hline History of smoking, n (\%) & $8(19.0)$ & $17(18.7)$ & 1.000 \\
\hline
\end{tabular}

Abbreviations: MAC-LD Mycobacterium avium complex lung disease, $B M I$ body mass index, IQR interquartile range

*Chronic diseases that required medication

${ }^{+}$Mann-Whitney $U$ test for continuous variables and Fisher's exact test for categorical variables

performed (Fig. 2). The AUC was 0.947 (95\% confidence interval $=0.899-0.995)$.

\section{Low serum E2 levels were related to MAC-LD even in age-} and BMI-matched subgroups

Among 42 patients with MAC-LD and 91 healthy controls, 17 patients with MAC-LD and 17 healthy controls were matched (Table 3). Even after age and BMI were matched, serum E2 $(2.05 \mathrm{pg} / \mathrm{mL}$ vs. $13.0 \mathrm{pg} / \mathrm{mL}, p<$ 0.001) was lower in MAC-LD patients than in healthy controls (Fig. 3a). ROC curve analysis showed that the
AUC of serum E2 was 0.917 (95\% confidence interval = 0.805-1.000) (Fig. 3b).

\section{Median E2 level of MAC-LD patients was lower than that of non-NTM BE patients}

We used 88 pooled samples of MAC-LD patients obtained from Sep 2015 to Jun 2018 and 18 pooled samples of non-NTM BE patients obtained from Nov 2017 to Oct 2018. Samples from 20 patients with MAC-LD and samples from 11 non-NTM BE patients were compared. Consistent with matching, we found that the median age ( 74 years vs 76 years, $p=0.699)$ and BMI $(18.98$

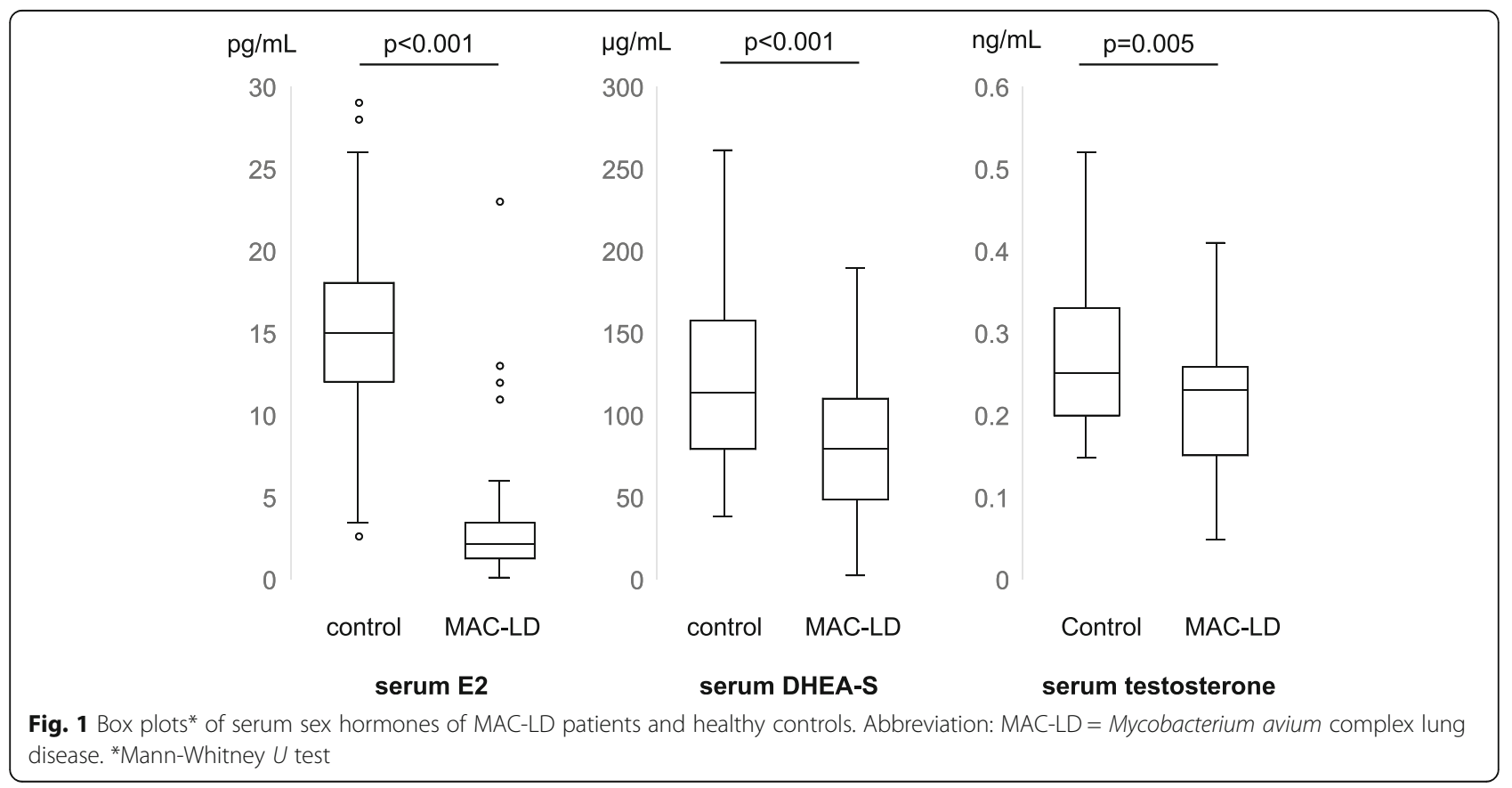


Table 2 Multivariate analysis of the relationships between MAC$\mathrm{LD}$ and age, BMI, and sex hormones

\begin{tabular}{lllll}
\hline & $\mathrm{OR}$ & $95 \% \mathrm{Cl}$ & adjusted OR & $95 \% \mathrm{Cl}$ \\
\hline Age & 1.42 & $1.25-1.62$ & 1.50 & $1.23-1.81$ \\
BMI & 0.77 & $0.67-0.89$ & 0.87 & $0.72-1.05$ \\
Low DHEA-S & 2.05 & $0.98-4.32$ & 1.17 & $0.35-3.95$ \\
Low testosterone & 1.78 & $0.85-3.72$ & 0.94 & $0.28-3.21$ \\
Low E2 & 52.80 & $11.87-234.95$ & 34.62 & $6.02-199.14$ \\
\hline
\end{tabular}

Abbreviations: MAC-LD Mycobacterium avium complex lung disease, OR odds ratio, $\mathrm{Cl}$ confidence interval, $B M I$ body mass index, DHEA-S

dehydroepiandrosterone sulfate, E2 estradiol

$\mathrm{kg} / \mathrm{m}^{2}$ vs $19.27 \mathrm{~kg} / \mathrm{m}^{2}, p=0.984$ ) of MAC-LD patients were equivalent to those of non-NTM BE patients. Median serum E2 levels in MAC-LD patients were significantly lower than median plasma E2 levels in non-NTM BE patients $(1.79 \mathrm{pg} / \mathrm{mL}$ vs. $11.0 \mathrm{pg} / \mathrm{mL}, p<0.001)$, while DHEA-S and testosterone were not significantly different between the two groups (Table 4).

\section{Discussion}

The incidence of pulmonary NTM infection has increased worldwide, and most cases are linked to MACLD. However, no effective treatment or preventive modality has been developed for MAC-LD because its pathogenesis is unclear. As most patients with MAC-LD are postmenopausal women, we speculated that low serum E2 levels represent a potential risk factor for MAC-LD. Our cross-sectional study revealed a link between low serum E2 levels and MAC-LD in

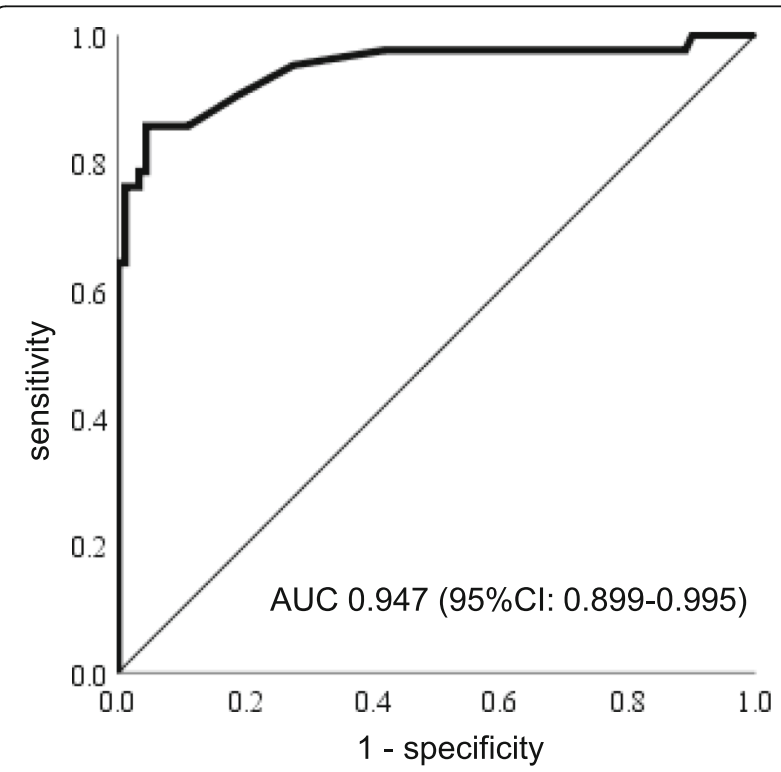

Fig. 2 ROC curve of estradiol serum levels in samples from MAC-LD patients and healthy controls. Abbreviations: $\mathrm{ROC}=$ receiver operating characteristics, MAC-LD = Mycobacterium avium complex lung disease, $\mathrm{AUC}=$ area under the curve, $\mathrm{Cl}=$ confidence interval postmenopausal women aged 65 years and younger. The age- and BMI-matched analyses also showed the same trends. Additionally, low serum E2 levels were seen in MAC but not in non-NTM BE, in women approximately 75 years-old, suggesting that low serum E2 was related specifically to MAC-LD.

Although we did not study causal relationships between low serum levels and MAC-LD, it is possible that E2 is protective against MAC infection. Tsuyuguchi et al. reported that ovariectomized mice had a higher MAC burden in the lungs 3-6 weeks after infection than sham-operated mice and E2-treated ovariectomized mice [10]. Calippe et al. found that long-term E2 administration to ovariectomized mice increased interleukin-1 beta secretion and inducible nitric oxide synthase expression which enhanced the killing of intracellular mycobacteria by resident peritoneal macrophages after lipopolysaccharide stimulation [11]. These reports indicate that E2 plays a protective role against MAC infection, in line with our findings. However, to demonstrate the protective role of E2 against MAC infection, further experimental studies are essential. For instance, the change in phagocytic and bactericidal abilities with or without E2 in human macrophages could be explored. Additionally, assessing the effects of changes in body weight, lung weight, and lung pathology using a MAC-infected aromatase-knockout mouse (which cannot produce E2 in either the ovary or peripherally) might reinforce our conclusion.

Danley et al. found that women with MAC-LD had lower serum DHEA-S levels but not lower serum estrogen levels than control subjects without lung disease [12]. However, our results identified an association between lower serum E2 levels and MAC-LD in postmenopausal women. Currently, we are unable to explain these divergent findings. To verify which sex hormone might affect host susceptibility to MAC-LD, additional research with larger sample size and age stratification is necessary. However, according to reports that DHEA is transformed to E2 by aromatase in macrophages, [13, 14] one could the hypothesize that both DHEA and estrogen have protective effects against MAC infection.

The ROC curve analysis suggested a strong association between MAC-LD and E2. Furthermore, the high AUC makes E2 a potential candidate in developing diagnostic tools. Although it is difficult to diagnose MAC-LD without microbiological findings and imaging, low E2 levels might indicate pretest probabilities of MAC-LD.

The findings of this study imply that controlling sex hormones could be a new approach for preventing or treating MAC-LD. Although additional research is required to uncover mechanisms and demonstrate causal relationships between low E2 levels and MAC-LD, measuring E2 and prescribing hormone replacement therapy 
Table 3 Demographic characteristics of age- and BMI-matched case controls

\begin{tabular}{|c|c|c|c|}
\hline & MAC-LD $(n=17)$ & Control $(n=17)$ & $p$-value ${ }^{\dagger}$ \\
\hline Age, years, median (IQR) & $59(58-60.5)$ & $59(58-60.5)$ & 1.000 \\
\hline BMI, median (IQR) & $19.35(17.81-20.80)$ & $20.36(19.00-21.08)$ & 0.394 \\
\hline Age at menopause, years, median (IQR) & $50(50-52)$ & $52(49-53)$ & 0.737 \\
\hline Age at first menstruation, years, median (IQR) & $12(11-13)$ & $12(11-13)$ & 1.000 \\
\hline Years after menopause, years, median (IQR) & $8(5-12)$ & $7(5-10.5)$ & 0.737 \\
\hline History of tuberculosis, n (\%) & $0(0.0)$ & $1(5.9)$ & 1.000 \\
\hline Chronic diseases, n (\%) & $4(23.5)$ & $7(41.2)$ & 0.465 \\
\hline Hypertension, $\mathrm{n}(\%)^{*}$ & $1(5.9)$ & $3(17.6)$ & 0.601 \\
\hline Diabetes mellitus, $n(\%)^{*}$ & $1(5.9)$ & $1(5.9)$ & 1.000 \\
\hline Dyslipidemia, n (\%)* & $2(11.8)$ & $4(23.5)$ & 0.656 \\
\hline Osteoporosis, n (\%)* & $1(5.9)$ & $1(5.9)$ & 1.000 \\
\hline History of smoking, n (\%) & $4(23.5)$ & $3(17.6)$ & 1.000 \\
\hline
\end{tabular}

Abbreviations: MAC-LD Mycobacterium avium complex pulmonary disease, $B M I$ body mass index, IQR interquartile range

*Chronic diseases that required medication

${ }^{\dagger}$ Mann-Whitney $U$ test for continuous variables and Fisher's exact test for categorical variables

for postmenopausal women with low E2 levels could be ancillary prevention and treatment option for MAC-LD patients.

The present study had several limitations. First, because the study sample was restricted to women no older than 65 years in the study comparison with healthy controls, the external validity of the study is limited. We needed healthy controls older than 65 years to solve this problem, however, it was slightly difficult to recruit a large number of "healthy" elderly women because the opportunity to access medical facilities of such a population were limited. We, therefore, included the additional study comparing MAC-LD with non-NTM BE, partially because this study population was approximately 75 years-old and demonstrated the same trends. Second, circadian rhythms might affect sex steroid hormone levels, especially that of testosterone, which is believed to be high in the morning and low in the evening. Third, it was uncertain whether low E2 levels were associated only with MAC-LD or also linked to chronic lung infections. To answer this question, further research comparing patients with MAC-LD to those with other chronic lung infections such as tuberculosis, aspergillosis or Mycobacterium abscessus lung infections and healthy

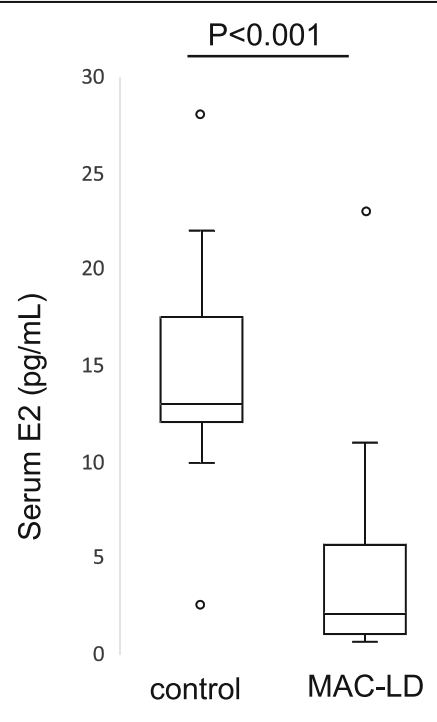

(a)

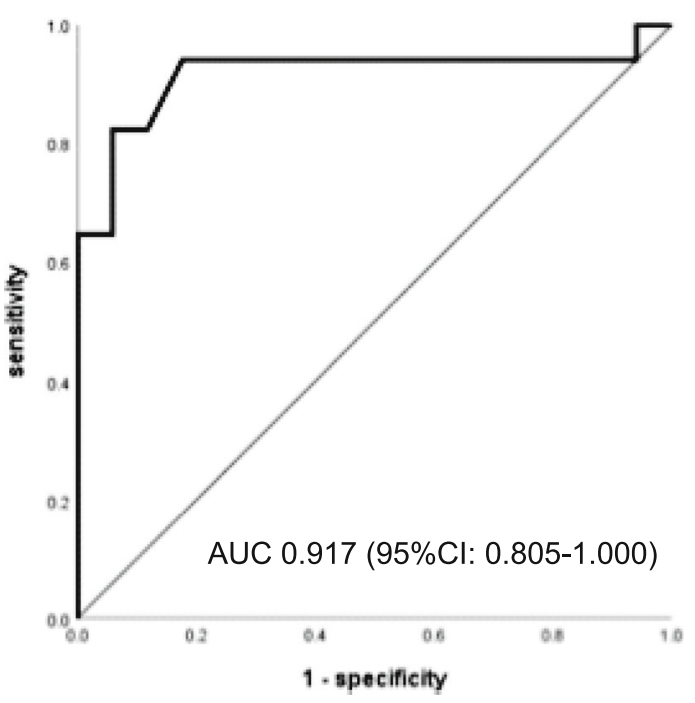

(b)

Fig. 3 Results of age- and BMI-matched subgroup analysis. a Box plot* of serum estradiol of age- and BMI-matched MAC-LD patients and healthy controls. b ROC curve of estradiol serum levels in samples from age- and BMI-matched MAC-LD patients and healthy controls. Abbreviations: $\mathrm{BMI}=$ body mass index, MAC-LD = Mycobacterium avium complex lung disease, $\mathrm{ROC}=$ receiver operating characteristics, $\mathrm{AUC}=$ area under the curve, $\mathrm{Cl}=$ confidence interval. *Mann-Whitney $U$ test 
Table 4 Demographic characteristics and sex hormone levels compared between patients with MAC-LD and non-NTM BE

\begin{tabular}{llll}
\hline & MAC-LD $(n=20)$ & Non-NTM BE $(n=11)$ & $p$-value \\
\hline Age, years, median (IQR) & $74(64.25-77.75)$ & $76(65-78)$ & 0.699 \\
BMl, median (IQR) & $18.98(17.65-20.97)$ & $19.27(17.29-21.62)$ & 0.984 \\
E2, pg/mL, median (IQR) & $1.79(1.20-2.47)$ & $11.0(2.77-13.0)$ & $<0.001$ \\
DHEA-S, $\mu \mathrm{g} / \mathrm{mL}$, median (IQR) & $51.5(39-97)$ & $85(53-99)$ & 0.145 \\
Testosterone, $\mathrm{ng} / \mathrm{mL}$, median (IQR) & $0.185(0.152-0.255)$ & $0.220(0.180-0.260)$ & 0.359 \\
\hline
\end{tabular}

Abbreviations: MAC-LD Mycobacterium avium complex lung disease, non-NTM BE bronchiectasis without non-tuberculous mycobacteria infection, BMI body mass index, IQR interquartile range

${ }^{*}$ Chronic diseases that required medication

${ }^{\dagger}$ Mann-Whitney $U$ test for continuous variables and Fisher's exact test for categorical variables

controls is necessary. Fourth, hormones measured in the non-NTM BE group were not from serum but plasma. We do not believe this affected our result because the difference between serum and plasma E2 levels is little according to previous studies measuring serum and plasma E2 at the same time $[15,16]$. Finally, it is unclear whether the decline in serum E2 levels precedes MACLD or whether MAC-LD reduces E2 concentrations. Future well-powered prospective and longitudinal studies may resolve these issues.

\section{Conclusion}

Low serum E2 levels were independently related to MAC-LD in middle-aged and presenile postmenopausal women.

\section{Abbreviations \\ AUC: area under the curve; BE: bronchiectasis; BMl: body mass index; CLIA: chemiluminescent immunoassay; DHEA-S: dehydroepiandrosterone sulfate; E2: estradiol; GPL: glycopeptidolipid; HIV: human immunodeficiency virus infection; Ig: immunoglobulin; MAC: Mycobacterium avium complex; MAC-LD: Mycobacterium avium complex lung disease; NTM: nontuberculous mycobacteria; ROC: receiver operating characteristic}

\section{Acknowledgments}

The authors thank the faculty and staff of Keio University who volunteered for this study.

\section{Authors' contributions \\ YU designed the study, collected and analyzed data and wrote the initial draft of the manuscript. TN and NH contributed to data collection, analysis and interpretation of data, and assisted in the preparation of the manuscript YS contributed to analysis and interpretation of data. TA and ET contributed to data collection and critically reviewed the manuscript. SU, MMo, $\mathrm{HF}, \mathrm{MI}$, $\mathrm{HK}$ and MMu contributed to data interpretation, and critically reviewed the manuscript.}

\section{Funding}

This study was supported by the Japan Society for the Promotion of Science (KAKENHI grant: 16 K19618 and 18 K16186) and Asteras Pharmaceutical Co. Ltd.

These funding bodies played no role in the design of the study and collection, analysis, and interpretation of data and in writing the manuscript.

\section{Availability of data and materials}

The datasets used and/or analyzed during the current study are available from the corresponding author on reasonable request.

\section{Ethics approval and consent to participate}

The study comparing MAC-LD patients with healthy controls was approved by the ethics committee at Keio University (No. 15-007) and the ethical committee of Keio University School of Medicine (No. 20150420). All participants provided written informed consent. The study comparing pooled samples of MAC-LD patients with those of non-NTM BE patients was approved by the ethical committee of Keio University School of Medicine (No. 20180261). All patients provided written informed consent when samples were originally obtained.

Consent for publication

Not applicable.

Competing interests

The authors declare that they have no competing interests.

\section{Author details}

${ }^{1}$ Department of Laboratory Medicine, Keio University School of Medicine, 35 Shinanomachi, Shinjuku-ku, Tokyo, Japan. ${ }^{2}$ Department of Infectious Diseases, Keio University School of Medicine, 35 Shinanomachi, Shinjuku-ku, Tokyo, Japan. ${ }^{3}$ Keio University Health Center, 35 Shinanomachi, Shinjuku-ku, Tokyo 160-8582, Japan. ${ }^{4}$ Department of Preventive Medicine and Public Health, Keio University School of Medicine, 35 Shinoanomachi, Shinjuku-ku, Tokyo, Japan. ${ }^{5}$ Division of Pulmonary Medicine, Department of Medicine, Keio University School of Medicine, 35 Shinanomachi, Shinjuku-ku, Tokyo, Japan. ${ }^{6}$ Department of Internal Medicine, Keio University School of Medicine, 35 Shinanomachi, Shinjuku-ku, Tokyo, Japan.

Received: 27 June 2019 Accepted: 29 November 2019

Published online: 16 December 2019

\section{References}

1. Adjemian J, Olivier KN, Seitz AE, Holland SM, Prevots DR. Prevalence of nontuberculous mycobacterial lung disease in U.S. Medicare beneficiaries. Am J Respir Crit Care Med. 2012;185(8):881-6.

2. Prevots DR, Marras TK. Epidemiology of human pulmonary infection with nontuberculous mycobacteria: a review. Clin Chest Med. 2015;36(1):13-4.

3. Namkoong H, Kurashima A, Morimoto K, Hoshino Y, Hasegawa N, Ato M, et al. Epidemiology of pulmonary nontuberculous mycobacterial disease, Japan. Emerg Infect Dis. 2016:22(6):1116-7.

4. Prince DS, Peterson DD, Steiner RM, Gottlieb JE, Scott R, Israel HL, et al. Infection with Mycobacterium avium complex in patients without predisposing conditions. N Engl J Med. 1989;321(13):863-8.

5. Chalermskulrat W, Gilbey JG, Donohue JF. Nontuberculous mycobacteria in women, young and old. Clin Chest Med. 2002;23(3):675-86.

6. Nishimura T, Fujita-Suzuki $Y$, Mori M, Carpenter SM, Fujiwara H, Uwamino $Y$, et al. Middle-aged to elderly women have a higher asymptomatic infection rate with Mycobacterium avium complex, regardless of body habitus. Respirology. 2016;21(3):553-5.

7. Griffith DE, Aksamit T, Brown-Elliott BA, Catanzaro A, Daley C, Gordin F, et al. An official ATS/IDSA statement: diagnosis, treatment, and prevention of nontuberculous mycobacterial diseases. Am J Respir Crit Care Med. 2007; 175(4):367-416.

8. Kitada S, Kobayashi K, Ichiyama S, Takakura S, Sakatani M, Suzuki K, et al. Serodiagnosis of Mycobacterium avium-complex pulmonary disease using an enzyme immunoassay kit. Am J Respir Crit Care Med. 2008;177(7):793-7. 
9. Kitada S, Levin A, Hiserote M, Harbeck RJ, Czaja CA, Huitt G, et al. Serodiagnosis of Mycobacterium avium complex pulmonary disease in the USA. Eur Respir J. 2013;42(2):454-60.

10. Tsuyuguchi K, Suzuki K, Matsumoto H, Tanaka E, Amitani R, Kuze F. Effect of oestrogen on Mycobacterium avium complex pulmonary infection in mice. Clin Exp Immunol. 2001;123(3):428-34.

11. Calippe B, Douin-Echinard V, Laffargue M, Laurell H, Rana-Poussine V, Pipy B, et al. Chronic estradiol administration in vivo promotes the proinflammatory response of macrophages to TLR4 activation: involvement of the phosphatidylinositol 3-kinase pathway. J Immunol. 2008;180(12):7980-8.

12. Danley J, Kwait R, Peterson DD, Sendecki J, Vaughn B, Nakisbendi K, et al. Normal estrogen, but low dehydroepiandrosterone levels, in women with pulmonary Mycobacterium avium complex. A preliminary study. Ann Am Thorac Soc. 2014;11 (6):908-14.

13. Mills SJ, Ashworth JJ, Gilliver SC, Hardman MJ, Ashcroft GS. The sex steroid precursor DHEA accelerates cutaneous wound healing via the estrogen receptors. J Invest Dermatol. 2005:125(5):1053-62.

14. Schmidt M, Kreutz M, Loffler G, Scholmerich J, Straub RH. Conversion of dehydroepiandrosterone to downstream steroid hormones in macrophages. J Endocrinol. 2000;164(2):161-9.

15. Fragalà S, Medica P, Grande F, Vazzana I, Fazio E. Evaluation of seasonal changes of serum and plasma estradiol-17\%, progesterone and testosterone in dolphins (Tursiops truncatus) by chemiluminescence. Vet World. 2015; 8(8):977-82.

16. Coburn SB, Stanczyk FZ, Falk RT, McGlynn KA, Brinton LA, Sampson J, et al. Comparability of serum, plasma, and urinary estrogen and estrogen metabolite measurements by sex and menopausal status. Cancer Causes Control. 2019:30(1):75-86.

\section{Publisher's Note}

Springer Nature remains neutral with regard to jurisdictional claims in published maps and institutional affiliations.

Ready to submit your research? Choose BMC and benefit from:

- fast, convenient online submission

- thorough peer review by experienced researchers in your field

- rapid publication on acceptance

- support for research data, including large and complex data types

- gold Open Access which fosters wider collaboration and increased citations

- maximum visibility for your research: over $100 \mathrm{M}$ website views per year

At $\mathrm{BMC}$, research is always in progress.

Learn more biomedcentral.com/submissions 